\title{
Metabolomics and Proteomics, and What to Do with All These 'Omes': Insights from Nutrigenomic Investigations in New Zealand
}

\author{
Matthew Barnett ${ }^{a} \quad$ Wayne Young ${ }^{a} \quad$ Janine Cooney ${ }^{d} \quad$ Nicole Roy ${ }^{a-c}$ \\ a Food Nutrition and Health Team, AgResearch Ltd., Grasslands Research Centre, and \\ ${ }^{b}$ Riddet Institute, Massey University, Palmerston North, ${ }^{\mathrm{c}}$ Gravida: National Centre for \\ Growth and Development, Auckland, and ${ }^{\mathrm{d}}$ Biological Chemistry and Bioactives, Plant \\ and Food Research Ltd., Ruakura Research Centre, Hamilton, New Zealand
}

\section{Key Words}

Systems biology $\cdot 1 l 10^{-/-}$mice $\cdot M d r 1 a^{-/-}$mice $\cdot$ Liquid chromatography-mass spectrometry

\begin{abstract}
Background/Aims: Nutrigenomics New Zealand has invested considerable effort researching the role of nutrient-gene interactions in human inflammatory bowel disease (IBD). This research has utilised a number of 'omics' techniques, including proteomics and metabolomics. Methods: Mouse models of intestinal inflammation have been used to investigate the mechanisms underlying IBD and to test foods or food components for potential beneficial effects. Proteomics combining two-dimensional gel electrophoresis with liquid chromatographymass spectrometry (LC-MS) analysis of peptides, and metabolomics using both gas chromatography-MS and LC-MS have been combined with transcriptomics and microbiome analyses to comprehensively assess samples derived from these models. Results: Across several independent studies, we have identified key proteins and metabolites which are involved in chronic inflammation. We have also identified food compounds such as polyphenols (green tea polyphenols or curcumin) and polyunsaturated fatty acids, or whole foods such as salmon and broccoli, that reduce inflammation by regulating the activity of these proteins and metabolites. Conclusions: Omics techniques, including proteomics and metabolomics, have deepened our insight into the mechanisms underlying intestinal inflammation, and how nutrientgene interactions may influence these. However, challenges remain in dealing with the enormous quantity of data generated by these techniques, and in utilising these data to improve the outcome for people with IBD.

(C) 2015 S. Karger AG, Basel
\end{abstract}

All authors are part of Nutrigenomics New Zealand (www.nutrigenomics.co.nz). This paper was presented at the 8th Congress of the International Society of Nutrigenetics/Nutrigenomics (ISNN), Gold Coast, Qld., Australia, May 2-3, 2014. 


\section{Introduction}

Nutrigenomics has been defined as the application of a range of the so-called omics techniques (including genomics, transcriptomics, proteomics, and metabolomics) to the field of human nutrition, especially the relationship between nutrition and health [1]. Nutrigenomics New Zealand (NuNZ; www.nutrigenomics.org.nz) is a collaboration between three New Zealand research organisations (AgResearch Ltd., Plant and Food Research, and The University of Auckland). Since 2004, NuNZ has applied nutrigenomics techniques to a variety of studies, using human inflammatory bowel disease (IBD) as a proof of concept. The long-term goal of the NuNZ programme 'Tailoring New Zealand Foods to Match People's Genes' is to develop foods that can be matched to individual human genotypes to benefit the health of specific genetic sub-groups. Over the course of its existence, NuNZ has completed numerous studies using mouse models of IBD, in which a range of omics techniques have been applied, including transcriptomics [2, 3], proteomics [4, 5], metabolomics [6], and analyses of the intestinal microbiota [7]. More recently, some of these techniques have been applied to samples derived from human dietary intervention studies investigating the anti-inflammatory potential of foods or diets $[8,9]$.

In this paper, we briefly describe some of the insights which the NuNZ programme has derived from the application of omics techniques, with a particular focus on proteomics and metabolomics. We also discuss when the application of these techniques is appropriate, as well as some of the issues associated with the use of omics technologies, in particular the very large amount of data generated and the application of developing methods to combine these large data sets to give a coherent view of a system as a whole. Finally, we consider whether there is an imbalance between the time, effort, and financial resource expended on the application of omics technologies and the development of tangible outcomes in terms of positive health impacts which are derived from omics data.

\section{When to Apply Omics Technologies}

Rapid advances in the development of omics technologies have led to their increasing application, both in general (fig. 1) and specifically in nutrition studies [1]. The large amounts of data such studies produce are a potential 'gold mine' of information for elucidating the effects of food and food ingredients on human health and well-being. However, the interpretation of these large data sets can represent a significant challenge.

In the case of the NuNZ programme, two key mouse models of IBD, the interleukin-10 gene-deficient $\left(1110^{-/-}\right)$mouse and the multi-drug resistance 1 gene-deficient $\left(\mathrm{Mdr}^{-\mathrm{a}^{-/-}}\right)$ mouse, have been used to investigate food components which may have a beneficial effect on intestinal inflammation. If a food component has been shown to prevent or reduce intestinal inflammation, then a range of omics technologies have been applied to gain insight into the mechanisms by which the food component exerts its effect.

Although the application of omics technologies is generally only thought appropriate if there are effects of a food on inflammation, some situations can warrant the use of these techniques in the absence of a clear phenotypic outcome. For example, NuNZ research has demonstrated that aqueous and ethyl acetate extracts of gold kiwifruit (Actinidia chinensis) or green kiwifruit (A. deliciosa) have anti-inflammatory activity in vitro, reducing inflammatory signalling in primary cells isolated from $I 110^{-/-}$mice [10]. These kiwifruit extracts were subsequently used in a dietary intervention study in $I l 10^{-/-}$mice, but they had no significant effect in reducing colonic inflammation, as assessed by histological examination [11]. Wholegenome gene and protein expression profiles were analysed to clarify this discrepancy 
Barnett et al.: Metabolomics and Proteomics, and What to Do with All These 'Omes': Insights from Nutrigenomic Investigations in New Zealand

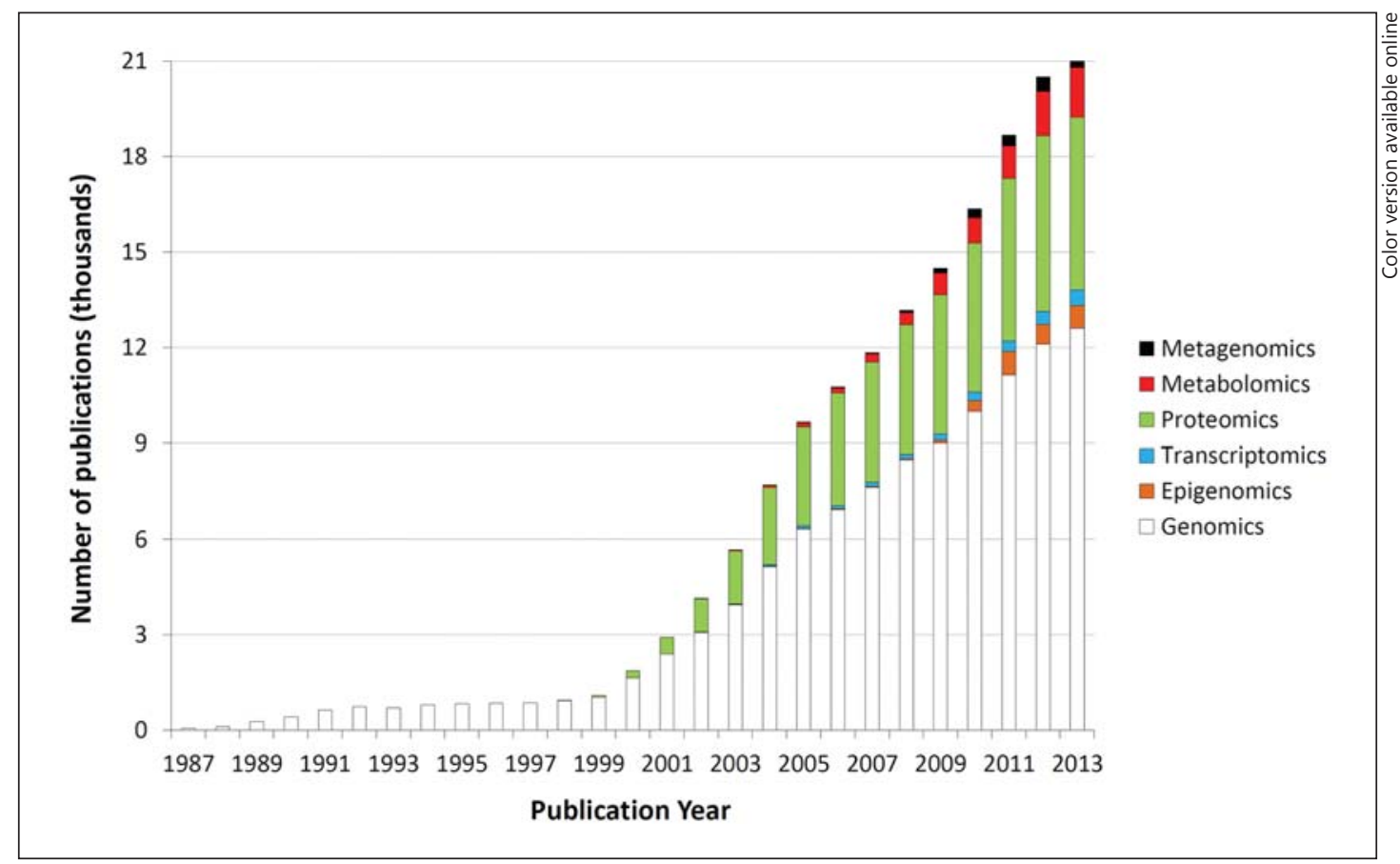

Fig. 1. Growing use of omics techniques. There is a rapid increase in the number of studies published in which a range of omics techniques (shown in the legend on the right-hand side) have been used. Data for this figure were obtained using a PubMed search (carried out in October 2014) using the terms shown in the legend, and the 'results by year' were then exported from PubMed to generate the figure. As an example, the search for the term 'metabolomics' returned a total of 8,073 results, ranging from 2 articles published in 2000 to 1,560 published in 2013. Results from 2014 were not included, because that calendar year was not complete at the time the search was performed.

between the two model systems. These analyses showed that while kiwifruit extracts influenced colon immune signalling pathways and metabolic processes, the effects were subtle and primarily related to adaptive immune pathways. This highlights that establishing a mechanistic understanding of discrepancies between in vitro and in vivo observations using omics technologies may help explain the limitations of in vitro models and the conditions under which they are appropriate or not appropriate to use. However, it must also be noted that the interpretation of results from omics approaches requires care, because the detection of false positives is likely due to the large amounts of data produced. Therefore, restraint needs to be exercised in the application of these methods to avoid 'throwing' omics technologies at a set of samples simply to generate data.

\section{Proteomics: One Genome, Two Proteomes}

The proteome is the set of all proteins produced by an organism or a system. The goal of proteomics has been described as 'a comprehensive, quantitative description of protein expression and its changes under the influence of biological perturbations' [12]. Thus, the study of proteomics comprehensively examines the protein composition and abundance in a given cell population. In the studies NuNZ has undertaken, we have used differential in-gel expression, in which samples from different treatment groups are labelled with different 


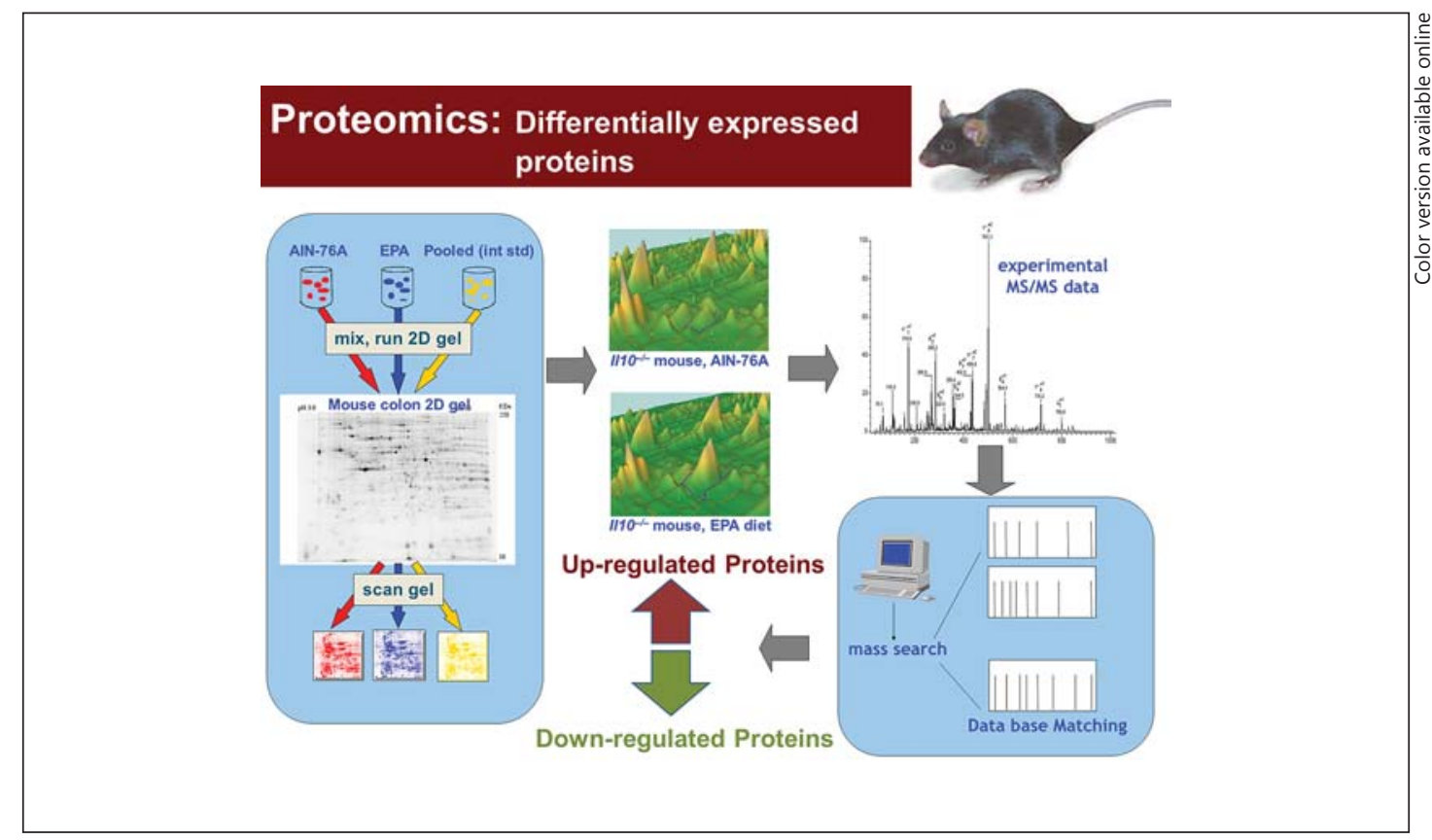

Fig. 2. The general approach used by NuNZ for proteomic analysis as applied to a mouse model of inflammatory bowel disease. After extracting the protein, samples from different treatment groups are labelled with Cy dyes, with a third dye used for a pooled sample as an internal standard. The labelled proteins are then subjected to 2D electrophoresis, and the gels are processed using image analysis to identify proteins which are expressed differentially between the treatment groups. In this case, the example of the $I 110^{-/-}$mouse fed a diet enriched with the n-3 polyunsaturated fatty acid eicosapentaenoic acid (EPA) is shown. Differentially expressed spots are then picked, and identified by LC-MS analysis of peptides derived by tryptic digestion. AIN-76A = Control diet.

cyanine (Cy) dyes (e.g. Cy2 for animals on a 'control' diet and Cy5 for those which have received a food treatment) and the proteins from both samples are separated on the same gel by 2D electrophoresis. Protein features which were differentially expressed between the treatments are then identified by liquid chromatography-mass spectrometry (LC-MS) analysis of peptides derived from the differentially expressed proteins.

These studies have focused on the effect of a nutritional intervention in mouse models of IBD, for example the effects of polyunsaturated fatty acids on inflammation in the $1110^{-/-}$ mouse [5], or the effect of polyphenols such as curcumin [13] or a polyphenol-rich green tea extract [4] on colon inflammation in the $M d r 1 a^{-/-}$mouse. A schematic of the general approach can be seen in figure 2 .

The key questions to be answered using this proteomics approach are:

- What proteins/pathways are associated with intestinal inflammation?

- What proteins/pathways are changed as a result of feeding a food component?

- Are these a potential target for a gene-specific food?

- How do these results relate to other omes?

Our studies to date have shown that inflammation in mouse models of IBD is associated with a general down-regulation of proteins involved with metabolism and digestion/ absorption/excretion of nutrients/ions, and up-regulation of cellular stress and immune response proteins. As already mentioned, nutritional intervention studies with polyunsaturated fatty acids and polyphenolics have shown their efficacy in reducing this inflammation and in modulating proteins associated with the inflammatory phenotype. We have also used 
Barnett et al.: Metabolomics and Proteomics, and What to Do with All These 'Omes':

Insights from Nutrigenomic Investigations in New Zealand

these data in combination with transcriptomics data and bioinformatics tools to identify modulated metabolic pathways and potential key gene/protein regulatory hubs that may regulate these processes $[4,5]$.

\section{Metabolomics}

Metabolomics 'aims to profile all the small molecule metabolites found within a cell, tissue, organ, or organism and use this information to understand a biological manipulation' [14]. Importantly, metabolomics analysis can be achieved using relatively non-invasive approaches, for example studying metabolites found in urine or plasma.

In the field of nutrigenomics, there are three major applications of metabolomics which are of particular relevance. The first application is to establish as complete a profile as possible of all compounds present in individual foods. This can be relevant when considering which nutrient or combination of nutrients might be contributing to a particular effect of the food in question.

The second important application is in the accurate assessment of what an individual has been eating. This is clearly of little relevance for animal studies, where the food intake of the animals can be strictly controlled. However, when studies are carried out in human populations, this can be of importance. Metabolite analysis to confirm that study participants are consuming the particular food or nutrient of interest better enables any observed beneficial effects to be linked to this nutrient. For studies in which complex foods or dietary interventions are being studied, assessment of a range of metabolites using a metabolomics approach can be applied. Figure $3 a$ and $b$ shows how this approach could be applied using the example of a dietary intervention in mice.

The third important application is identifying metabolites associated with disease. This is of particular relevance within the context of biomarkers, i.e. identifying molecules which can act as prognostic or diagnostic markers of disease. These may also enable the effective monitoring of an intervention - although their development is not without challenges, as will be discussed later.

As shown in figure 3, metabolomics data sets can be used to identify overall effects, for example using principal components, principal co-ordinate, or partial least squares discriminant analyses, which look at the overall pattern of metabolites for an individual (fig. 3a, b). They can also be interrogated in detail to identify more specific effects relating to individual metabolites across a data set (fig. 3c).

Similarly to proteomics, metabolomics aims to answer some key questions regarding changes in the levels of a metabolite (or pattern of metabolites), such as:

- Does a metabolite correlate with the degree of inflammation?

- Does it predict the onset of inflammation or predict remission?

- Does it therefore allow effective monitoring of health status or of the impact of a nutrient intervention?

\section{Integration of Omics Data}

A major challenge is the integration of data from the various omics technologies to obtain biologically relevant information. This may be to accurately define a phenotype, to better understand the complexity of the biological response to specific bioactive food components, or to identify key food-responsive molecular targets for disease prevention or health maintenance. Software packages such as Ingenuity Pathway Analysis have been developed to inte- 
Barnett et al.: Metabolomics and Proteomics, and What to Do with All These 'Omes': Insights from Nutrigenomic Investigations in New Zealand

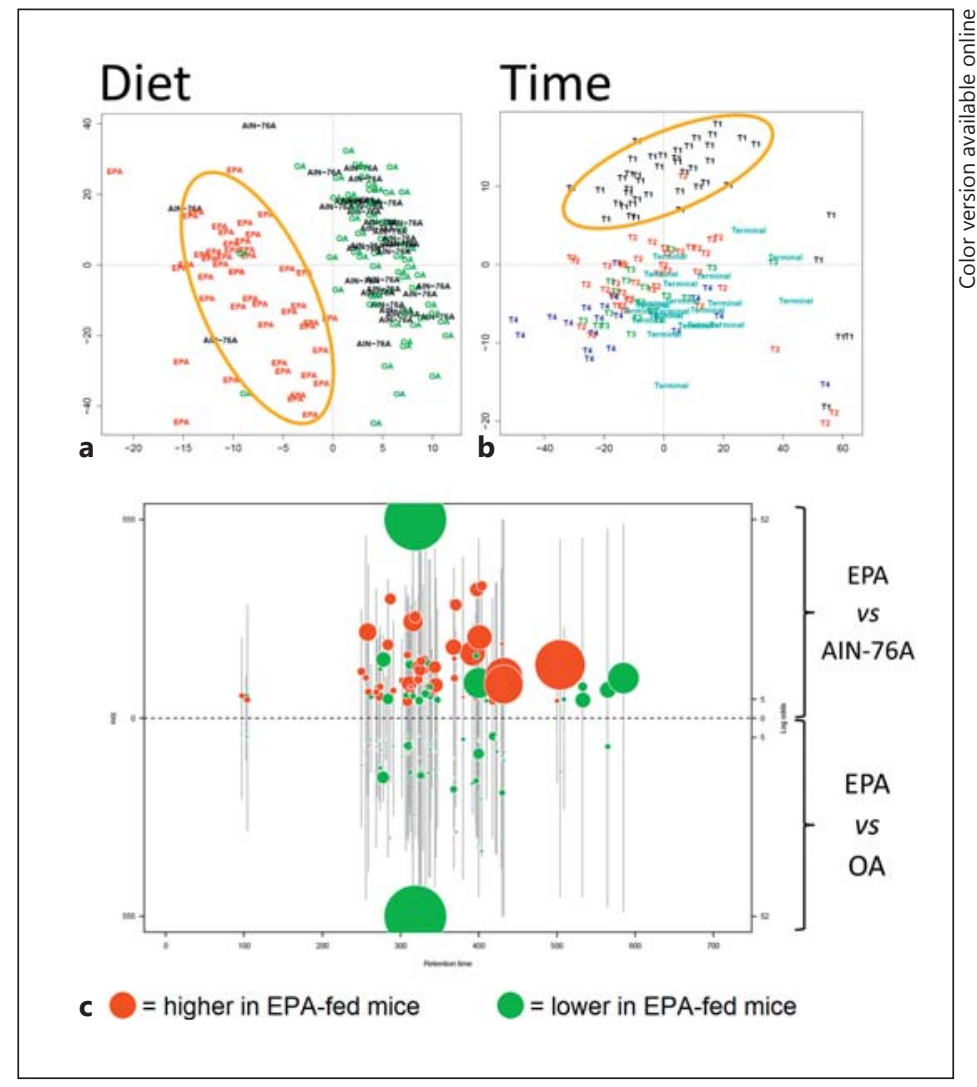

Fig. 3. Approaches to representing metabolomics data. Partial least squares discriminant analysis has been used to distinguish the urine metabolomics profiles from individual $I l 10^{-/-}$mice based on diet (a) and sampling time (b) [21]. c 'Cloud plot' [22] to visualise global MS-based metabolomics data, in which differences in individual metabolites due to dietary intervention can be seen. In this plot, metabolites that have significantly different signal intensities between mice fed eicosapentaenoic acid (EPA)-enriched or control (AIN76A) diets are shown in the upper half [21], while metabolites that different between mice fed oleic acid (OA)- and EPA-enriched diets are shown in the lower half. Metabolites with higher signal intensities in response to the EPA diet are coloured red (colours refer to the online version only), while those that are lower in EPA-fed mice are coloured green. The size of the circle is proportional to fold change, and the distance from the $\mathrm{x}$-axis indicates the significance of fold change, with values further from 0 indicating greater significance.

grate omics data from a variety of experimental platforms and to analyse, interpret, and visualise changes in genes and proteins (as an example) according to biological pathways and networks. Functional analysis of this type enables key insights into experimental data by identifying relationships, mechanisms, functions, and pathways of relevance, and it can predict the cause and effects of changes in gene expression through transcription factor activation or inhibition. A number of other tools have been developed which address this challenge, attempting to integrate these large data sets using so-called systems biology approaches.

In the case of NuNZ, we are using some of these tools to try and answer questions such as:

- Does a metabolite/protein/mRNA/miRNA/histone modification (or pattern of these) correlate with the degree of intestinal inflammation?

- Does it predict the onset of inflammation or predict remission?

- Does it allow effective monitoring of health status?

- Does it demonstrate a beneficial effect of a food, which could therefore represent a potential 'gene-specific' food with potential for providing beneficial effects for IBD? 


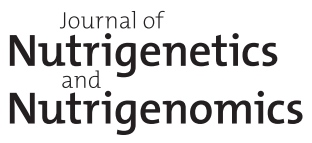

Fig. 4. Application of omics techniques in biomarker discovery. Omics technologies are primarily applied during the discovery phase of developing a biomarker. An apparent lack of effective translation to the subsequent qualification and application phases may in part be explained by an over-use of omics technologies, leading to a subsequent 'discovery loop'. Figure adapted from Koulman et al. [19].

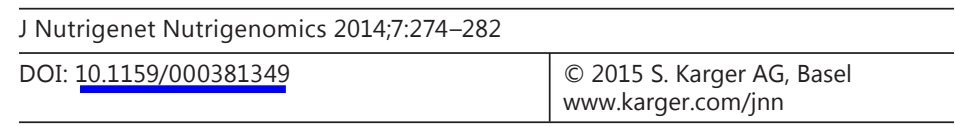

Barnett et al.: Metabolomics and Proteomics, and What to Do with All These 'Omes': Insights from Nutrigenomic Investigations in New Zealand

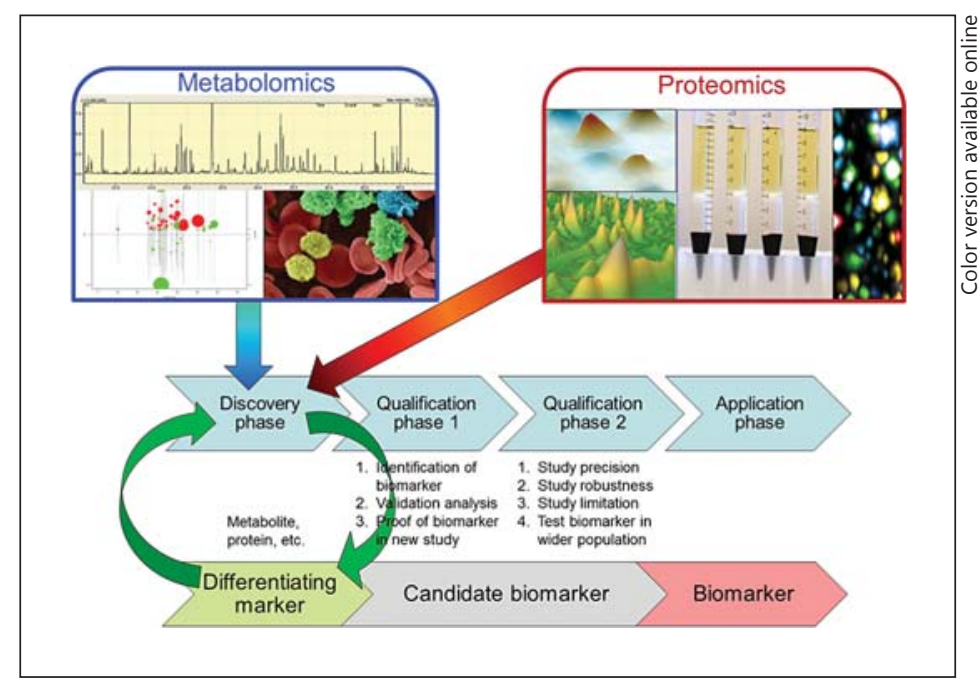

An argument could be made that a combination of omics data (systems biology) is necessary to identify robust markers of 'health' and to comprehensively establish gene-specific effects of food components. Ideally, integration of data from these different omics platforms will lead to a more comprehensive picture of the processes responsible for particular phenotypes, a picture that would not be possible by analysing the different omics data in isolation. One example to briefly illustrate this point is that of acetaminophen, more commonly known as paracetamol, which is detoxified in the liver by the addition of sulphate [15]. While this process is well understood, there is considerable variation in the efficiency of this process from person to person, some of which can be explained by different genetic polymorphisms in genes involved in acetaminophen detoxification [16]. However, it is now also understood that $p$-cresol produced by the intestinal microbiota competes with acetaminophen for sulphation in the liver [17]. Therefore, the efficiency of acetaminophen detoxification can be explained as a function both of host and microbial activity. Without the integrated analysis of omics data from both the host and the microbiota, this relationship could not have been elucidated. In another example of successful multi-omics analyses, a novel alkaloid metabolic pathway was discovered in Podophyllum hexandrum through the integration of RNA sequencing and comprehensive metabolomics analyses [18]. This example also demonstrates the potential importance of combining large-scale data sets to better understand complex biological systems.

\section{Finding Early Biomarkers of Disease}

As already mentioned, one important potential application of omics technologies such as metabolomics, but also proteomics and transcriptomics, is in the discovery, development, and qualification of biomarkers. Omics techniques are primarily contributing to the discovery phase of biomarker development - looking at overall patterns without necessarily having an a priori hypothesis of what changes are likely to occur, with the goal of identifying metabolites, proteins, or other molecules which may be diagnostic or prognostic. The relative paucity of effective translation of fundamental omics research into practical dietary, clinical, or therapeutic outcomes may not be surprising, given that the post-discovery phases of this process may be long and require significant work [19]. However, it perhaps also begs the question: is over-use of omics keeping us stuck in the 'discovery' phase (fig. 4)? Analysis of samples using 
omics will almost certainly lead to a large amount of data, and there may be an understandable temptation to generate more data, rather than - as Rifai et al. [20] so eloquently put it - start down 'the long and uncertain path to clinical utility'.

\section{Conclusions}

There is clearly an important role for the various omics technologies in nutrition research. Whether investigating the key mechanisms through which a food may exert its functional effects, defining a phenotype with very high accuracy and sensitivity, or understanding how an intervention can be monitored, the generation and integration of these large-scale data sets help to provide a comprehensive overall view of a biological system. With changing regulatory requirements for making health claims for a food, including evidence for its mode of action, investigations combining omics data are likely to become more important in nutrition research. However, as with the case of biomarker discovery, omics techniques tend to be most relevant at the early stages of this research. Although they provide a large amount of data, these can be essentially descriptive and do not necessarily provide mechanistic evidence of cause and effect, for which carefully designed follow-up studies are necessary.

One example of a desirable long-term outcome would be in the application of a combination of particular foods or food components with a selection of sensitive phenotypic biomarkers for the treatment of disease/optimisation of health. For example, a food for which there is robust evidence of a beneficial effect on intestinal health could form part of a treatment or management regime, with an appropriate panel of gene/protein/metabolite biomarkers being used to accurately and, importantly, non-invasively assess the impact of this regime. However, there appears to be a lack of effective translation of fundamental omics research into tangible outcomes or interventions at the clinical, therapeutic, or dietary level. This is a challenge that can, and must, be addressed if omics technologies such as metabolomics and proteomics are to fulfil their undoubted potential.

\section{Disclosure Statement}

Some of the work described here was part of the Nutrigenomics New Zealand programme, a collaboration between AgResearch Ltd., Plant and Food Research Ltd., and The University of Auckland; this collaboration was largely funded by the New Zealand Ministry of Business, Innovation and Employment (contract No. C02X0403 and C11X1009). The authors declare no conflict of interest.

\section{References}

1 Fenech M, El-Sohemy A, Cahill L, Ferguson LR, French TA, Tai ES, Milner J, Koh WP, Xie L, Zucker M, Buckley M, Cosgrove L, Lockett T, Fung KY, Head R: Nutrigenetics and nutrigenomics: viewpoints on the current status and applications in nutrition research and practice. J Nutrigenet Nutrigenomics 2011;4:69-89.

-2 Barnett MP, McNabb WC, Cookson AL, Zhu S, Davy M, Knoch B, Nones K, Hodgkinson AJ, Roy NC: Changes in colon gene expression associated with increased colon inflammation in interleukin-10 gene-deficient mice inoculated with Enterococcus species. BMC Immunol 2010;11:39.

-3 Knoch B, Barnett MP, McNabb WC, Zhu S, Park ZA, Khan A, Roy NC: Dietary arachidonic acid-mediated effects on colon inflammation using transcriptome analysis. Mol Nutr Food Res 2010;54(suppl 1):S62-S74.

-4 Barnett MP, Cooney JM, Dommels YE, Nones K, Brewster DT, Park Z, Butts CA, McNabb WC, Laing WA, Roy NC:

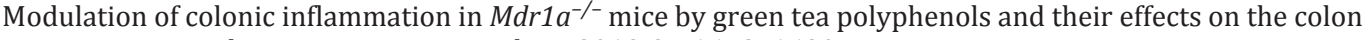
transcriptome and proteome. J Nutr Biochem 2013;24:1678-1690.

5 Cooney JM, Barnett MP, Brewster D, Knoch B, McNabb WC, Laing WA, Roy NC: Proteomic analysis of colon tissue from interleukin-10 gene-deficient mice fed polyunsaturated fatty acids with comparison to transcriptomic analysis. J Proteome Res 2012;11:1065-1077. 


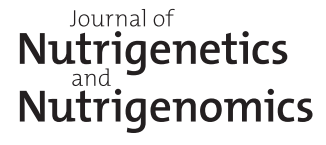

Nutrigenetics

Nutrigenomics

-6 Lin HM, Barnett MP, Roy NC, Joyce NI, Zhu S, Armstrong K, Helsby NA, Ferguson LR, Rowan DD: Metabolomic analysis identifies inflammatory and noninflammatory metabolic effects of genetic modification in a mouse model of Crohn's disease. J Proteome Res 2010;9:1965-1975.

7 Knoch B, Nones K, Barnett MP, McNabb WC, Roy NC: Diversity of caecal bacteria is altered in interleukin-10 gene-deficient mice before and after colitis onset and when fed polyunsaturated fatty acids. Microbiology 2010;156:3306-3316.

-8 Ellett S, Ferguson IR, Zhu S, Karunasinghe N, Marlow G, Hurley D, Lam WJ, Han DY, Ferguson LR: Foodomics to study efficacy of human dietary interventions; in Ferguson LR (ed): Nutrigenomics and Nutrigenetics in Functional Foods and Personalized Nutrition. Boca Raton, CRC Press, 2013, pp 269-280.

-9 Marlow G, Ellett S, Ferguson IR, Zhu S, Karunasinghe N, Jesuthasan AC, Han DY, Fraser AG, Ferguson LR: Transcriptomics to study the effect of a Mediterranean-inspired diet on inflammation in Crohn's disease patients. Hum Genomics 2013; 7:24.

10 Edmunds SJ, Roy NC, Love DR, Laing WA: Kiwifruit extracts inhibit cytokine production by lipopolysaccharideactivated macrophages, and intestinal epithelial cells isolated from IL10 gene deficient mice. Cell Immunol 2011;270:70-79.

-11 Edmunds SJ, Roy NC, Davy M, Cooney JM, Barnett MP, Zhu S, Park Z, Love DR, Laing WA: Effects of kiwifruit extracts on colonic gene and protein expression levels in IL-10 gene-deficient mice. Br J Nutr 2012;108:113129.

-12 Anderson NL, Anderson NG: Proteome and proteomics: new technologies, new concepts, and new words. Electrophoresis 1998;19:1853-1861.

13 Nones K, Dommels YE, Martell S, Butts C, McNabb WC, Park ZA, Zhu S, Hedderley D, Barnett MP, Roy NC: The effects of dietary curcumin and rutin on colonic inflammation and gene expression in multidrug resistance gene-deficient ( $\mathrm{mdr}^{-/-}$) mice, a model of inflammatory bowel diseases. Br J Nutr 2009;101:169-181.

$>14$ Griffin JL, Vidal-Puig A: Current challenges in metabolomics for diabetes research: a vital functional genomic tool or just a ploy for gaining funding? Physiol Genomics 2008;34:1-5.

15 Lee S, Dawson PA, Hewavitharana AK, Shaw PN, Markovich D: Disruption of NaS1 sulfate transport function in mice leads to enhanced acetaminophen-induced hepatotoxicity. Hepatology 2006;43:1241-1247.

-16 Court MH, Freytsis M, Wang X, Peter I, Guillemette C, Hazarika S, Duan SX, Greenblatt DJ, Lee WM; Acute Liver Failure Study Group: The UDP-glucuronosyltransferase (UGT) 1A polymorphism c.2042C >G (rs8330) is associated with increased human liver acetaminophen glucuronidation, increased UGT1A exon 5a/5b splice variant mRNA ratio, and decreased risk of unintentional acetaminophen-induced acute liver failure. J Pharmacol Exp Ther 2013;345:297-307.

-17 Clayton TA, Baker D, Lindon JC, Everett JR, Nicholson JK: Pharmacometabonomic identification of a significant host-microbiome metabolic interaction affecting human drug metabolism. Proc Natl Acad Sci USA 2009;106: 14728-14733.

18 Marques JV, Dalisay DS, Yang H, Lee C, Davin LB, Lewis NG: A multi-omics strategy resolves the elusive nature of alkaloids in Podophyllum species. Mol Biosyst 2014;10:2838-2849.

19 Koulman A, Lane GA, Harrison SJ, Volmer DA: From differentiating metabolites to biomarkers. Anal Bioanal Chem 2009;394:663-670.

20 Rifai N, Gillette MA, Carr SA: Protein biomarker discovery and validation: the long and uncertain path to clinical utility. Nat Biotechnol 2006;24:971-983.

21 Barnett MPG, Young W, Otter DE, Roy NC: Metabolomics and gene expression analyses of the Il10 gene-deficient mouse model of IBD; in NuGO Week 2013. Freising-Weihenstephan, Wageningen Academic Publishers, 2013, p 81.

-22 Patti GJ, Tautenhahn R, Rinehart D, Cho K, Shriver LP, Manchester M, Nikolskiy I, Johnson CH, Mahieu NG, Siuzdak G: A view from above: cloud plots to visualize global metabolomic data. Anal Chem 2013;85:798-804. 